\title{
Effect of Heat and Mass Transfer on Unsteady MHD Oscillatory Flow of Jeffrey Fluid in a Horizontal Channel with Chemical Reaction
}

\author{
IDOWU, A.S ${ }^{1}$.JOSEPH, K. $\mathrm{M}^{1}$. and DANIEL, $\mathrm{S}^{2}$. \\ 1 Department of Mathematics, University of Ilorin, Ilorin, Nigeria \\ 2. Department of Mathematics, Kaduna State University, Kaduna, Nigeria
}

\begin{abstract}
The effect of heat transfer on unsteady MHD oscillatory flow of Jeffrey fluid in a horizontal channel with chemical reaction has been studied. The temperature prescribed at plates is uniform and asymmetric. A perturbation method is employed to solve the momentum and energy equations. The skin frictions, the Nusselt numbers and Sherwood are evaluated using perturbation technique. The effects of various dimensionless parameters on velocity and temperature profiles are considered and discussed in details through graphs and tables. It is found that, the velocity u increases with increase in $G r, G c, N$ and Re. The velocity also increases with decrease in $\lambda_{1}, \mathrm{Ha}, \mathrm{Sc}$ and $\omega$. The velocity only decreases with increase in Pe. It is also observed that the temperature $\theta$ decreases with increase in $N$, Re and $\omega$. Decrease in Schmidt number Sc, chemical parameter Kc and frequency of oscillation $\omega$ increase the species concentration or the concentration boundary layer thickness of the flow field.

Keywords: Heat Transfer, Unsteady, MHD, Jeffrey Fluid, Oscillatory Flow
\end{abstract}

\section{Introduction}

The effect of heat transfer on unsteady MHD oscillatory flow of fluid in horizontal media are encountered in a wide range of engineering and industrial applications such as molten iron flow, recovery extraction of crude oil, geothermal systems. Many chemical engineering processes like metallurgical and polymer extrusion processes involve cooling of a molten liquid being stretched in a cooling system; the fluid mechanical properties of penultimate product depend mainly on the cooling liquid used and the rate of stretching. Some polymers fluids like polyethylene oxide and polysobutylene solutions in a cetane, having better electromagnetic properties are normally used as cooling liquid as their flow can be regulated by external magnetic fields in order to improve the quality of the final product. Also, the radiative heat transfers is an important factor of thermodynamics of very high temperature systems such as electric furnaces, solar collectors, storage of nuclear wastes packed bed catalytic reactors, satellites, steel rolling, cryogenic engineering etc.

The study of such flow under the influence of magnetic field and heat transfer has attracted the interest of many investigators and researchers.

Asadullah et al (2013) consider the MHD flow of a Jeffrey fluid in converging and diverging channels. The flows between non parallel walls have a very significant role in physical and biological sciences.

Kavita et al (2012) investigated the influence of heat transfer on MHD oscillatory flow of Jeffery fluid in a Channel. An analysis of first order homogeneous chemical reaction and heat source on MHD oscillatory flow of viscous - elastic fluid through a channel filled with saturated porous medium are reported by Devika et al (2013)

Siddiqui et al (2013) made an attempt to study the classical Von Karma flow problem for Jeffrey fluid by using a generalized non - similarity transformation.

An oscillatory flow of a Jeffrey Fluid in an elastic tube of variable cross - section has been investigated at low Reynolds number by Badari et al (2012). Their main concentration is on the excess pressure of the tube. The equation has been solved numerically and investigations are made for different cases on the tube.

Aruna Kumari et al (2012) studied the effect of heat transfer on MHD oscillatory flow of Jeffrey fluid in a channel with slip effect at a lower wall where the expressions for the velocity and temperature are obtained analytically.

Unsteady Flow of a Jeffrey Fluid in an elastic tube with stenosis was investigated by Sreenadh et al (2012). The governing equation for the excess pressure is obtained for Jeffrey model. The governing equations has been solved numerically and investigations are made for different cases for strength,tapered and constricted tubes.

Isreal - Cookey et al (2010) investigated the combined effects of radiative heat transfer and a transverse magnetic field on steady flow of an electrically conducting optically thin fluid through a horizontal channel 
filled with porous medium and non - unform temperatures at the walls. Closed form analytical solutions for the problem.

The unsteady MHD free convective flow through porous medium sandwiched between electrically conducting viscous - incompressible fluids in a horizontal channel with isothermal walls temperature using Brinkman model has been investigated by Kumar et al (2012).

The forced convection in a horizontal double - passage with uniform wall heat flux has been studied by Joseph et al (2013) by taking into account the effect of magnetic parameter where the flow of the fluid is assumed to be laminar, two - dimensional, steady and fully developed. The fluid is incompressible and the physical properties are constants and the walls are kept at uniform heat flux.

Bodosa and Borkati (2003) analysed the problem of an unsteady two - dimensional flow of a viscous incompressible and electrically conducting fluid between two parallel plates in the presence of uniform transverse magnetic field.

Choudhury and Das (2013) presented an analysis of unsteady MHD flow of an electrically conducting visco elastic fluid confined between two horizontal parallel no - conducting in presence of a transverse magnetic field and Hall current. The lower plate is a stretched sheet while the upper one is an oscillating porous plate, which is oscillating in its own plane.

The effect of radiation on unsteady free convection flow bounded by an oscillating plate with variable wall temperature was studied by Pathak and Maheshwari (2006).

The flow and heat transfer between two horizontal parallel plates, where the lower plate is a stretching sheet and the upper one is a porous solid plate in the presence of transverse magnetic field was discussed by Borkakati and Bharali (1980). Also Bharali and Borkakati (1983) studied the heat transfer in an axisymmetric flow between two parallel porous disk under the effect of a transverse magnetic field.

Sharma and Deka (2012) studied the effects of plate temperature oscillation on the unsteady conducting fluid along a semi - infinite vertical porous plate subjected to a transverse magnetic field in the presence of a first order chemical reaction and thermal radiation.

Al - Rashdan (2012) presented an analytical investigation to the problem of fully - developed natural convective heat mass transfer through porous medium in a channel in the presence of a first order - order chemical reaction.

The effect of magnetic field on free convective flow of a viscous incompressible fluid past an infinite moving porous hot vertical plate in the presence of porous medium and radiation was analyzed by Singh et al (2012).

$\mathrm{El}$ - Hakiem et al (2000) studied the MHD oscillatory flow on free convection radiation through a porous medium with constant velocity. Singh et al (2011) investigated the numerical steady of two - dimensional MHD forward stagnation point of flow in the presence of hall current.

Isreal - Cookey and Nwaigwe (2010) studied the unsteady MHD flow of a radiating fluid over a moving heated plate with time - dependent suction. The MHD free convective flow of viscous fluid through a porous through a medium bounded by an oscillating porous plate in slip flow regime with mass transfer was studied by Singh and Gupta (2005).

Kim (2000) discussed the unsteady MHD convective heat transfer past a semi - infinite vertical porous moving plate with variable suction. The problem of unsteady MHD periodic flow of viscous fluid through a planar channel in porous medium using perturbation techniques was considered by Kumar et al (2010).

Makinde and Mhone (2005) studied the heat transfer to MHD oscillatory flow in a channel filled with porous medium.

This present paper focused on the effect of heat transfer on unsteady MHD oscillatory flow of fluid in a horizontal infinite parallel plate channel. The fluid considered is Jeffrey Fluid. Perturbation technique was employed to obtain the expressions for the velocity and temperature profiles. The graphs and tables were used to discuss in detail the effects of various emerging parameters on the velocity and temperature.

\section{Problem Formulation}

We consider the flow of Jeffrey fluid in horizontal infinite parallel plates channel. The channel width is $h$. The constitute equation of for $S$ Jeffrey fluid Kavita et al (2012) is

$S=\frac{\mu}{1+\lambda_{1}}\left(\frac{d \eta}{d t^{\prime}}+\lambda_{2} \frac{d^{2} \eta}{d t^{\prime 2}}\right)$

Where $\mu$ is the dynamic viscosity, $\lambda_{1}$ is the ratio of relaxation to retardation times, $\lambda_{2}$ is the retardation time and $\frac{d \eta}{d t}$ is the shear rate.

Now, let $u^{\prime}$ be the velocity of the fluid in $x^{\prime}$ direction taken along the horizontal infinite parallel plate channel under a chemical reaction with species concentration $C^{\prime}$ and $y^{\prime}-$ axis is taken normal to the direction of flow. The radiative heat term in $x^{\prime}$ direction is considered negligible in comparison with $y^{\prime}$ direction. The upper and lower plates are kept at $T^{\prime}=T_{\infty}^{\prime}$ and $T^{\prime}=T_{w}^{\prime}$ respectively. The Cartesian coordinate system and the flow configuration are shown in figure 1 . At a time $t^{\prime}>0$, the plate is given an impulsive motion in a horizontal 
direction with uniform mean velocity $U$. Moreover at this stage an unsteady component $\varepsilon T^{\prime} e^{i w t^{\prime}}$ where $\varepsilon \ll 1$ is the amplitude of oscillation, is assumed to be superimposed on the temperatures of the plates.

$$
T^{\prime}=T_{\infty}^{\prime}
$$
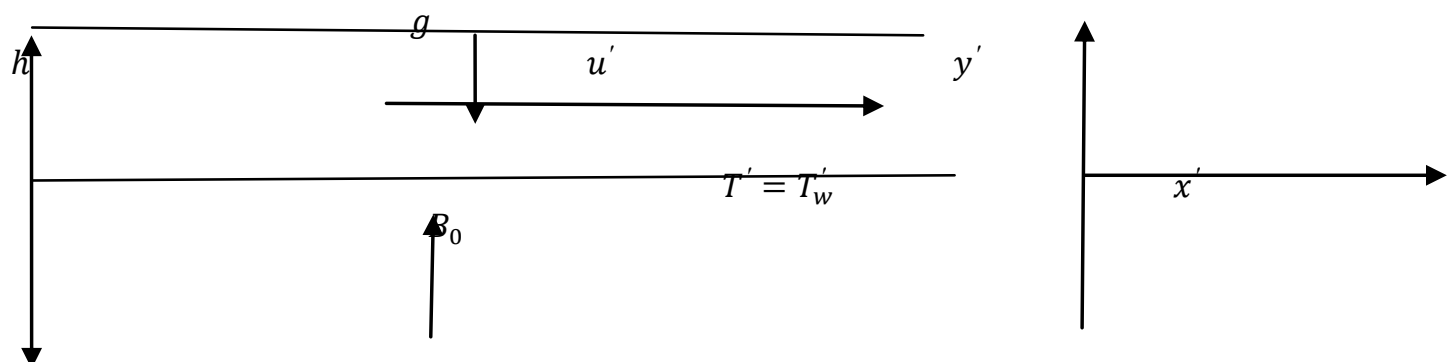

Figure 1. Physical schematic of the flow configuration

A magnetic field of uniform strength $B_{0}$ is applied normal to the plate along $x^{\prime}$ direction and the induced magnetic field is assumed negligible. It is also assumed that the fluid has small electrical conductivity and the electromagnetic force produced is very small. The pressure gradient is also assumed negligible. Since the plate is considered infinite in $x^{\prime}$ direction, all the physical quantities will be independent of $x^{\prime}$. Under these assumptions, the physical variables are functions of $y^{\prime}$ and $t^{\prime}$ only.

The basic equations of momentum and energy govern such a flow, subject to Boussinesq approximation, are

$\frac{\partial u^{\prime}}{\partial t^{\prime}}=-\frac{\partial p}{\partial x^{\prime}}+\frac{\mu}{\rho\left(1+\lambda_{1}\right)} \frac{\partial^{2} u^{\prime}}{\partial y^{\prime 2}}-\frac{\sigma B_{0} u^{\prime}}{\rho}+g \beta\left(T^{\prime}-T_{\infty}^{\prime}\right)+g \beta^{\prime}\left(C^{\prime}-C_{\infty}^{\prime}\right)$

$\frac{\partial T^{\prime}}{\partial t^{\prime}}=\frac{k}{\rho c_{p}} \frac{\partial^{2} T^{\prime}}{\partial y^{\prime 2}}-\frac{1}{\rho c_{p}} \frac{\partial q}{\partial y^{\prime}}$

$\frac{\partial C^{\prime}}{\partial t^{\prime}}=D \frac{\partial^{2} C^{\prime}}{\partial y^{\prime 2}}-K_{c}^{\prime}\left(C^{\prime}-C_{\infty}^{\prime}\right)$

The $q$ in (2.3) is called the radiative heat flux. It is given by

$\frac{\partial q}{\partial y^{\prime}}=4 \alpha^{2}\left(T_{w}^{\prime}-T^{\prime}\right)$

Where, $u^{\prime}$ is the axial velocity, $T^{\prime}$ is the fluid temperature, $\rho$ is the fluid density, $\lambda_{1}$ is the ratio of relaxation to retardation times, $B_{0}$ is the magnetic field strength, $\sigma$ is the conductivity of the fluid, $g$ is the acceleration due to gravity, $\beta$ is the coefficient of volume expansion due to temperature, $c_{p}$ is the specific heat at constant pressure, $k$ is the thermal conductivity, $D$ is the mass diffusion coefficient, $K_{c}$ is the chemical reaction parameter.

The relevant boundary conditions are given by

$u^{\prime}=0, \quad T^{\prime}=T_{w}^{\prime}, C^{\prime}=C_{w}^{\prime} \quad$ at $y^{\prime}=0, t^{\prime} \leq 0$

$u^{\prime}=0, \quad T^{\prime}=T_{\infty}^{\prime}, C^{\prime}=C_{\infty}^{\prime} \quad$ at $y^{\prime}=h, t^{\prime} \geq 0$

$u^{\prime} \rightarrow 0, \quad T^{\prime} \rightarrow 0, \quad C^{\prime} \rightarrow 0 \quad$ as $y^{\prime}=0, \forall t^{\prime}$

In order to write the governing equations and the relevant boundary conditions in non - dimensional form, the following dimensionless quantities are introduced

$x=\frac{x^{\prime}}{h}, \quad y=\frac{y^{\prime}}{h}, \quad u=\frac{u^{\prime}}{U}, \quad \theta=\frac{T^{\prime}-T_{w}^{\prime}}{T_{\infty}^{\prime}-T_{w}^{\prime}}, \quad t=\frac{t^{\prime} U}{h}, \quad H a^{2}=\frac{\sigma h^{2} B_{0}^{2}}{\mu}, \quad \quad G r=\frac{\rho h^{2} g \beta\left(T_{\infty}^{\prime}-T_{w}^{\prime}\right)}{\mu U}$,

$R e=\frac{\rho h U}{\mu}, P e=\frac{\rho h U c_{p}}{k}, N^{2}=\frac{4 \alpha^{2} h^{2}}{k}, K_{c}=\frac{K_{c}^{\prime} v}{U}$,

$S c=\frac{U}{D}, G c=\frac{\rho h^{2} \beta^{\prime}\left(C_{\infty}^{\prime}-C_{w}^{\prime}\right)}{\mu U}$

The momentum equation (2.2), the energy equation (2.3) and the species concentration equation (2.4) now become

$R e \frac{\partial u}{\partial t}=\frac{1}{1+\lambda_{1}} \frac{\partial^{2} u}{\partial y^{2}}-H a^{2} u+G r \theta$

$P e \frac{\partial \theta}{\partial t}=\frac{\partial^{2} \theta}{\partial y^{2}}-N^{2} \theta$

$\frac{\partial C}{\partial t}=\frac{1}{S c} \frac{\partial^{2} C}{\partial y^{2}}-K_{c} C$

The relevant boundary conditions in dimensionless form are

$u=0, \quad \theta=0, \quad C=0 \quad$ at $y=0, t \leq 0$

$u=0, \quad \theta=1, \quad C=1 \quad$ at $y=0, t \geq 0$

$u \rightarrow 0, \quad \theta \rightarrow 0, \quad C \rightarrow 0 \quad$ at $y \rightarrow \infty, \forall t$

\section{Solution Of The Problem}

To solve equations $(2.7)-(2.8)$ subject to the boundary conditions (2.9), we expand the functions $u(y, t)$ and $\theta(y, t)$ as a power series in the perturbative parameter $\varepsilon$. Here we assumed small amplitude of oscillations $(\varepsilon \ll 1)$, thus 
$u(y, t)=u_{0}(y)+\varepsilon u_{1}(y) e^{i \omega t}+o\left(\varepsilon^{2}\right)$

$\theta(y, t)=\theta_{0}(y)+\varepsilon \theta_{1}(y) e^{i \omega t}+o\left(\varepsilon^{2}\right)$

$C(y, t)=C_{0}(y)+\varepsilon C_{1}(y) e^{i \omega t}+o\left(\varepsilon^{2}\right)$

The first terms in equations (3.1), (3.2) and (3.3) are called the harmonic terms while the second terms are called the non - harmonic terms.

Substituting equations (3.1), (3.2) and (3.3) into equations (2.8), (2.9) and (2.10), equating the coefficients of the harmonic and non - harmonic terms and neglecting the coefficients of $\varepsilon^{2}$, we get:

$a u_{0}^{\prime \prime}(y)-H a^{2} u_{0}(y)=-G r \theta_{0}(y)-G c C_{0}(y)$

$a u_{1}^{\prime \prime}(y)-b^{2} u_{1}(y)=-\operatorname{Gr} \theta_{1}(y)-G c C_{1}(y)$

Where, $a=\frac{1}{1+\lambda_{1}}$ and $b^{2}=H a^{2}+i \omega R e$

$\theta_{0}^{\prime \prime}(y)-N^{2} \theta_{0}(y)=0$

$\theta_{1}^{\prime \prime}(y)-c^{2} \theta_{1}(y)=0$

Where, $c^{2}=N^{2}+i \omega P e$

$C_{0}^{\prime \prime}(y)-b_{2} C_{0}(y)=0$

$C_{1}^{\prime \prime}(y)-d C_{1}(y)=0$

Where, $b_{2}=K_{c} S c$ and $d=i w S c+K_{c} S c$

The corresponding relevant boundary conditions become

$u_{0}=0, \theta_{0}=0, C_{0}=0$ at $y=0, t \leq 0$

$u_{1}=0, \theta_{1}=1, C_{1}=1$ at $y=0, t \geq 0$

$u_{0} \rightarrow 0, \theta_{0} \rightarrow 0, C_{0} \rightarrow 0$ at $y \rightarrow \infty, \forall t$

$u_{1} \rightarrow 0, \theta_{1} \rightarrow 0, C_{1} \rightarrow 0$ at $y \rightarrow \infty, \forall t$

We now solved equations (3.4) - (3.9) under the relevant boundary conditions (3.10) for the mean flow and unsteady flow separately.

The mean flows are governed by the equations (3.4), (3.6) and (3.8) where $u_{0}, \theta_{0}, C_{0}$ are respectively called the mean velocity, mean temperature and mean species concentration. The unsteady flows are governed by equations (3.5), (3.7) and (3.9) where $u_{1}, \theta_{1}$ and $C_{1}$ are the unsteady components.

These equations are solved analytically under the relevant boundary conditions (3.10) as follows;

Solving equations (3.6) and (3.8) subject to the corresponding relevant boundary conditions in (3.10), we obtain the mean temperature as

$\theta_{0}(y)=\frac{\sinh N y}{\sinh N}$

$C_{0}(y)=\frac{\sinh \sqrt{b_{2}} y}{\sinh \sqrt{b_{2}}}$

Similarly, solving equations (3.7) and (3.9) under the relevant boundary conditions in (3.10), the unsteady temperature becomes

$\theta_{1}(y)=2 \operatorname{sinnsinhcy}$

Where, $c=\sqrt{N^{2}+i \omega P e}$

$C_{1}(y)=2 \operatorname{sinm} \sinh \sqrt{d} y$

Where, $d=i w S c+K_{c} S c$

Putting equations (3.11), (3.12) and equations (3.13) and (3.14) into equations (3.4) and (3.5) respectively and using the corresponding boundary conditions in (3.10), we obtain the mean velocity $u_{0}(y)$ and the unsteady velocity component $u_{1}(y)$ as follows;

$u_{0}(y)=2\left(\frac{G r A \sinh N}{E \sinh D}+\frac{G c B \sinh \sqrt{b_{2}}}{F \sinh D}\right) \sinh D y-\frac{2 G r A \operatorname{sinhN} y}{E}-\frac{2 G c B \sinh \sqrt{b_{2} y}}{F}$

And

$u_{1}(y)=2\left(\frac{g G r \sinh c}{J \sinh G}+\frac{G c H \sinh \sqrt{d}}{L \sinh G}\right) \sinh G y-\frac{2 g G r \sinh c y}{J}-\frac{2 G c H \sinh \sqrt{d} y}{L}$

Therefore, the solutions for the velocity and temperature profiles are $u(y, t)=$

$2\left(\left(\frac{G r A \sinh N}{E \sinh D}+\frac{G \operatorname{cBsinh} \sqrt{b_{2}}}{F \sinh D}\right) \sinh D y-\frac{2 G r A \operatorname{sinhN} y}{E}-\frac{2 G \operatorname{GB\operatorname {sinh}} \sqrt{b_{2}} y}{F}\right)+\varepsilon\left[2\left(\frac{g G r \sinh c}{J \sinh G}+\frac{G \operatorname{Gr} \sinh \sqrt{d}}{L \sinh G}\right) \sinh y-\right.$ $\left.\frac{2 g \text { Grsinhcy }}{J}-\frac{2 G c H \sinh \sqrt{d} y}{L}\right] e^{i \omega t}$

$\theta(y, t)=\frac{\sinh N y}{\sinh N}+\varepsilon[2 \operatorname{sinn} \pi \sinh c y] e^{i \omega t}$

$C(y, t)=\frac{\sinh \sqrt{b_{2}} y}{\sinh \sqrt{b_{2}}}+\varepsilon[2 \operatorname{sinm} \sinh \sqrt{d} y] e^{i \omega t}$

Where, $\quad a=\frac{1}{1+\lambda_{1}} \quad b^{2}=H a^{2}+i \omega R e$ 


$$
\begin{array}{lr}
b_{2}=K_{c} S c & c=\sqrt{N^{2}+i \omega P e} \\
d=i w S c+K_{c} S c & A \\
B=\frac{1}{e^{\sqrt{b_{2}}}-e^{-\sqrt{b_{2}}}} & \\
E=a N^{2}-H a^{2} & F \\
g_{1}=\operatorname{sinn} \pi & G=\frac{b}{\sqrt{a}} \\
H=\operatorname{sinm} \pi & J=a c^{2}- \\
L=a d-b^{2} &
\end{array}
$$

\section{Skin Friction $\tau$}

The skin friction $\tau$ is given by

$$
\tau=-\mu \frac{d u^{\prime}}{d y^{\prime}}
$$

Here, we considered two skin frictions $\tau_{0}$ and $\tau_{1}$ at $y=0$ and $y=1$ respectively.

And in view of equation (2.10), equation (3.21) becomes

$$
\tau_{0}=-\frac{\rho U^{2}}{R e} \frac{d u}{d y}=\left.\frac{d u}{d y}\right|_{y=0}=\left.\frac{d u_{0}}{d y}\right|_{y=0}+\left.\varepsilon e^{i \omega t} \frac{d u_{1}}{d y}\right|_{y=0}
$$

Finding $\left.\frac{d u_{0}}{d y}\right|_{y=0}$ and $\left.\frac{d u_{1}}{d y}\right|_{y=0}$ from equation (3.15) and substituting into equation (3.22) and simplifying, we get the required skin friction $\tau_{0}$ as

$\tau_{0}=$

$2 D\left(\frac{G r A \sinh N}{E \sinh D}+\frac{G c B \sinh \sqrt{b_{2}}}{F \sinh D}\right)-\frac{2 N G r A}{E}-\frac{2 \sqrt{b_{2}} G c B}{F}+\varepsilon\left[2 G\left(\frac{g_{1} G r \sinh c}{J \sinh G}+\frac{G c H \sinh \sqrt{d}}{L \sinh G}\right)-\frac{2 c g_{1 G r}}{J}-\frac{2 \sqrt{a} G c H}{L}\right] e^{i \omega t}$

Where $a, b, b_{2}, c, d, g_{1}, A, B, D, E, F, G, H, J$ and $L$ are defined in equation (3.20)

Similarly, the skin friction $\tau_{1}$ is also given as

$\tau_{1}=-\frac{\rho U^{2}}{R e} \frac{d u}{d y}=\left.\frac{d u}{d y}\right|_{y=1}=\left.\frac{d u_{0}}{d y}\right|_{y=1}+\left.\varepsilon e^{i \omega t} \frac{d u_{1}}{d y}\right|_{y=1}$

Which on finding $\left.\frac{d u_{0}}{d y}\right|_{y=1}$ and $\left.\frac{d u_{1}}{d y}\right|_{y=1}$ from equation (3.16) and substituting into equation (3.24) and simplifying, we get the required skin friction $\tau_{1}$ as

$\tau_{1}=$

$2 D\left(\frac{G r A \sinh N}{E \sinh D}+\frac{G c B \sinh \sqrt{b_{2}}}{F \sinh D}\right) \cosh D-\frac{2 N G r A \cosh N}{E}-\frac{2 \sqrt{b_{2}} G c B \cosh \sqrt{b_{2}}}{F}+\varepsilon\left[2 G\left(\frac{g_{1} G r \sinh c}{J \sinh G}+\frac{G c H \sinh \sqrt{d}}{L \sinh G}\right) \cosh G-\right.$

$\left.\frac{2 c g_{1} \operatorname{rrcosh} c}{J}-\frac{2 \sqrt{d} G \cosh \sqrt{d}}{L}\right] e^{i \omega t}$

Where $a, b, b_{2}, c, d, g_{1}, A, B, D, E, F, G, H, J$ and $L$ are defined in equation (3.20)

\section{The Nusselt Number $\mathrm{Nu}$}

This is the coefficient of heat transfer.

We also consider the Nusselt numbers namely $N u_{0}$ and $N u_{1}$ at the two boundaries i.e. at $y=0$ and $y=1$ respectively.

$N u_{0}=\left.\frac{d \theta_{0}}{d y}\right|_{y=0}+\left.\frac{d \theta_{1}}{d y}\right|_{y=0}$

Finding $\left.\frac{d \theta_{0}}{d y}\right|_{y=0}$ and $\left.\frac{d \theta_{1}}{d y}\right|_{y=0}$ from equations (3.11) and (3.13) and substituting into equation (3.26) and simplifying, we get the required Nusselt number $N u_{0}$ as

$N u_{0}=\frac{N}{2 \sinh N}+2 \varepsilon c E e^{i \omega t}$

Where, $c$ and $E$ are defined in equation (3.20)

Similarly, the Nusselt number $N u_{1}$ at $y=1$ is given by

$N u_{0}=\left.\frac{d \theta_{0}}{d y}\right|_{y=1}+\left.\frac{d \theta_{1}}{d y}\right|_{y=1}$

Which on finding $\left.\frac{d \theta_{0}}{d y}\right|_{y=1}$ and $\left.\frac{d \theta_{1}}{d y}\right|_{y=1}$ from equations (3.11) and (3.13) and substituting into equation (3.20) and simplifying, we get the required Nusselt number $N u_{1}$ as

$N u_{1}=\frac{N}{\sinh N} \cosh N+\varepsilon(2 c E \cosh c) e^{i \omega t}$

Where $c$ and $E$ are defined in equation (3.20)

\section{Sherwood number $S h$}

This is the coefficient of chemical reaction.

We also consider the Sherwood numbers namely $S h_{0}$ and $S h_{1}$ at the two boundaries i.e. at $y=0$ and $y=1$ respectively. 
$S h_{0}=\left.\frac{d C_{0}}{d y}\right|_{y=0}+\left.\frac{d C_{1}}{d y}\right|_{y=0}$

Finding $\left.\frac{d C_{0}}{d y}\right|_{y=0}$ and $\left.\frac{d C_{1}}{d y}\right|_{y=0}$ from equations (3.12) and (3.14) and substituting into equation (3.30) and simplifying, we get the required Sherwood number $S h_{0}$ as

$S h_{0}=\frac{\sqrt{b_{2}}}{\sinh \sqrt{b_{2}}}+2 \varepsilon \sqrt{d} H e^{i \omega t}$

Where, $b_{2}, d$ and $H$ are defined in equation (3.20)

Similarly, the Sherwood number $S h_{1}$ at $y=1$ is given by

$S h_{1}=\left.\frac{d C_{0}}{d y}\right|_{y=1}+\left.\frac{d C_{1}}{d y}\right|_{y=1}$

Which on finding $\left.\frac{d C_{0}}{d y}\right|_{y=1}$ and $\left.\frac{d C_{1}}{d y}\right|_{y=1}$ from equations (3.12) and (3.14) and substituting into equation (3.32) and simplifying, we get the required Sherwood number $S h_{1}$ as

$S h_{1}=\frac{\sqrt{b_{2}} \cosh \sqrt{b_{2}}}{\sinh \sqrt{b_{2}}}+\varepsilon(2 \sqrt{d} H \cosh \sqrt{d}) e^{i \omega t}$

Where $b_{2}, d$ and $H$ are defined in equation (3.20)

\section{Analysis and Discussion of Results}

In this section, 2 - term perturbation series are employed to evaluate the dimensionless velocity and dimensionless temperature profiles. The radius of convergence of the perturbation series can be easily be obtained by estimating the value of the D'Alembert ratio test (1984).

To study the effect of heat transfer on unsteady MHD oscillatory flow of a Non - Newtonian Fluid (Jeffrey Fluid) in a horizontal infinite parallel plates channel, the velocity $u$ and the temperature $\theta$ profiles are depicted graphically against $y$ for different values of different parameters; material parameter $\lambda_{1}$, Grashof numbers Gr and Gc, Hartman number $\mathrm{Ha}$, Reynolds number Re, radiation parameter $N$, Schmidt number $S c$, chemical reaction parameter $K_{c}$, frequency of oscillation $\omega$, and Peclet number $P e$.

The velocity of the flow field varies to a great extend with the variation of the flow parameters. The main factors affecting the velocity are $\lambda_{1}, G r, G c, H a, R e, N, S c, K c, \omega$ and $P e$. The effects of these parameters on the velocity flow field are analyzed in table1 and figures 2-10. These effects are discussed quantitatively.

Figure 2 demonstrates the effect of material parameter $\lambda_{1}$ on velocity $u$. It is evident that the velocity $u$ increases with decrease in $\lambda_{1}$. To this effect the ratio of relaxation to retardation enhances the increase flow of velocity when it becomes small.

Figure 3 shows the effect of Grashof number $G r$ on velocity $u$. It is observed that as $G r$ increases, the velocity increases. To this effect, at higher Grashof number $\mathrm{Gr}$ the flow at the boundary is turbulent while at lower $\mathrm{Gr}$ the flow at the boundary is laminar.

The effect of the radiation parameter $N$ on velocity $u$ is depicted in figure 4 . It is observed that the velocity $u$ increases as the radiation parameter $N$ decreases.

Figure 5 depicts the effect of Hartman number $H a$ on velocity $u$. It is shown that the velocity $u$ increases with decrease in $\mathrm{Ha}$. This shows the effect of magnetic field on the fluid flow and this effect suppresses the turbulence flow of the Jeffrey fluid. Physically, when magnetic field is applied to any fluid, then the apparent viscosity of the fluid increases to the point of becoming visco elastic solid. It is of great interest that, yield stress of the fluid can be controlled very accurately through variation of magnetic field intensity. The result is that the ability of the fluid to transmit force can be controlled with the help of electromagnet which give rise to many possible control-based applications, including MHD power generation, electromagnetic casting of metals, MHD ion propulsion, etc.

Figure 6 illustrates the effect of frequency of oscillation $\omega$ on velocity $u$. It is observed that the velocity $u$ increases with decrease in the frequency of oscillation.

The effect of Reynolds number $R e$ on velocity $u$ is shown in figure 7. It is shown that the velocity $u$ increases with increasing Re.

Figure 8 depicts the effect of Grashof number $G c$ for mass transfer on velocityu. It is observed that the velocity $u$ increases with increase in $G c$.

Figure 9 demonstrate the effect of Schmidt number $S c$ on velocity $u$. It shows that the velocity increases with decrease in $S c$.

Figure 10 illustrates the effect of chemical reaction parameter $K_{c}$ on velocity $u$.it is observed that as the chemical reaction parameter decreases, the velocity increases. The lines of the graphs in figure 2 to figure 10 , converge at the points $y=0$ and $y=1$, this is simply because the points are the boundary conditions for the velocity $u$. The expression for $u$ must satisfied the conditions. Also, since the flow of the fluid is oscillatory, the graphs for velocity $u$ clearly shown a pure oscillation. 
The temperature field suffers a major change in magnitude due to the variation of parameters $\mathrm{N}, \mathrm{Re}$ and $\omega$. The effects of these parameters on temperature field are discussed in figures 11-13, which shows retardation in the magnitude of temperature field at all point.

Figure 11 depicts the effect of radiation parameter $N$ on temperature $\theta$. It is found out the temperature decreases with increase in $N$.

The effect of Reynolds number $R e$ on temperature $\theta$ is shown in figure 12. It is observed that the temperature $\theta$ decreases with increase in $R e$.

Figure 13 reveals the effect of frequency of oscillation $\omega$ on temperature $\theta$. It is revealed that the temperature $\theta$ decreases with increase in $\omega$.

The presence of foreign mass in the flow field greatly affects the spices concentration of the flow field, these parameters include $S c, K c$ and $\omega$.

The effect of Schmidt number $S c$ on species concentration $C$ is shown in figure 14. It is shown that the species concentration $C$ increases with decrease in $S c$.

Figure 15 reveals the effect of chemical reaction $K_{c}$ on species concentration $C$. It is observed that decrease in $K_{c}$ increases the species concentration $C$.

The effect of angular velocity $\omega$ on species concentration $C$ is depicted in figure 16. It is shown that the species concentration $C$ increases with decrease in $\omega$.

Table 1 shows the variation of velocity $u$ for different values of Peclet number $P e$. It shown that, the velocity $u$ decreases with increase in $P e$.

Tables 2, 3 and 4 respectively show the numerical values of skin frictions $\left(\tau_{0}\right.$ and $\left.\tau_{1}\right)$, Nusselt numbers $\left(N u_{0}\right.$ and $\left.N u_{1}\right)$ and Sherwood numbers $\left(S h_{0}\right.$ and $\left.S h_{1}\right)$. These numerical values are obtained by means of $2-$ term perturbation series for different values of time $t$. It is shown in figure 2 that the skin friction $\tau_{0}$ decreases with increase in time $t$ while $\tau_{1}$ increases as time $t$ increases. Table 3 shows that both $N u_{0}$ and $N u_{1}$ decreases as time $t$ increase. Table 4 shows that both the $S h_{0}$ and $S h_{1}$ decreases with increase in time $t$.

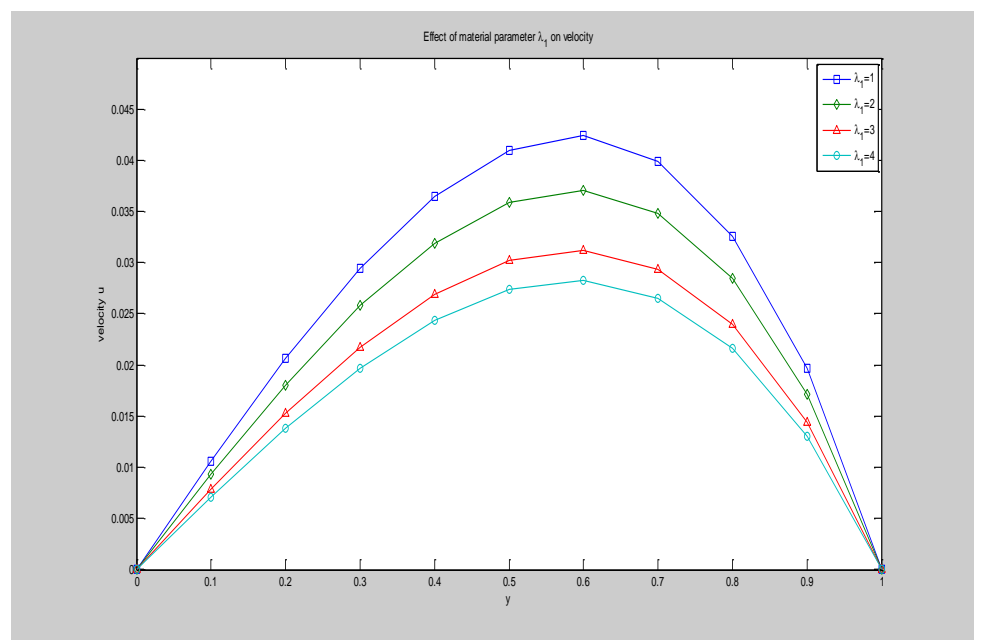

Figure 2.

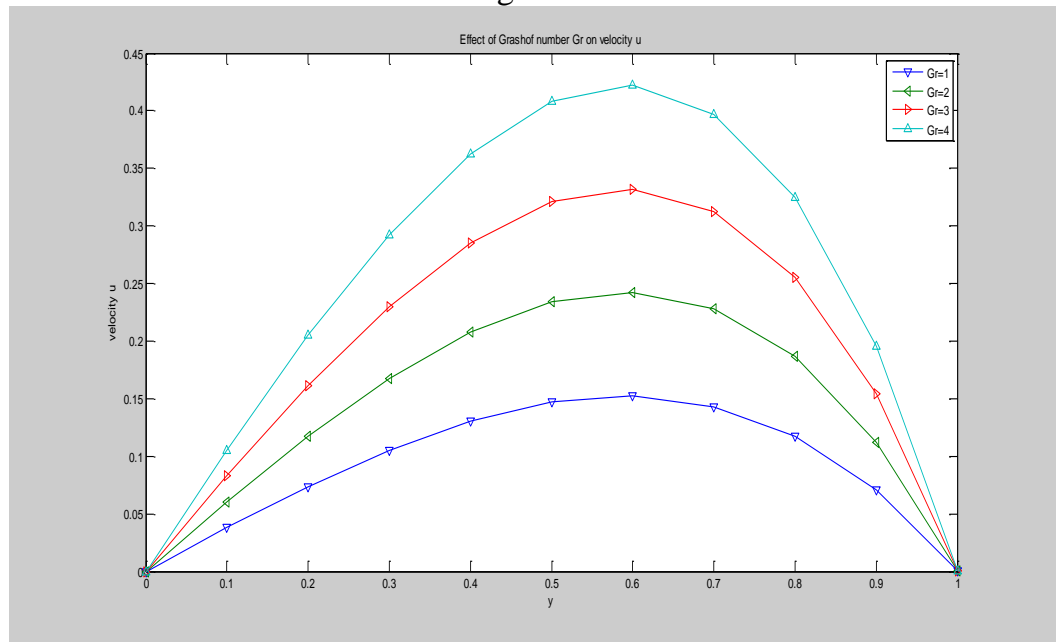

Figure 3. 


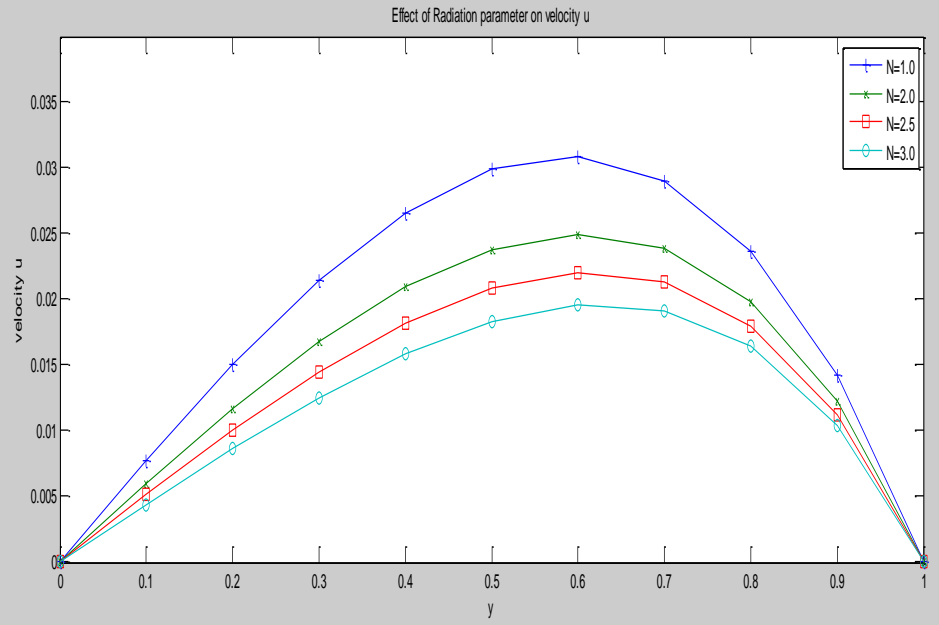

Figure 4.

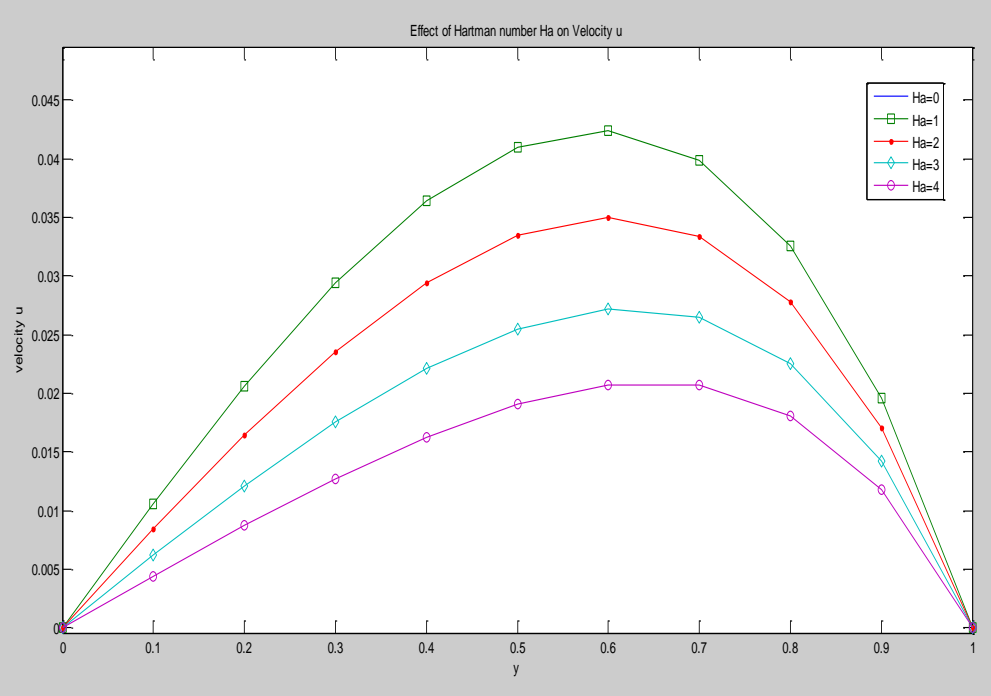

Figure 5.

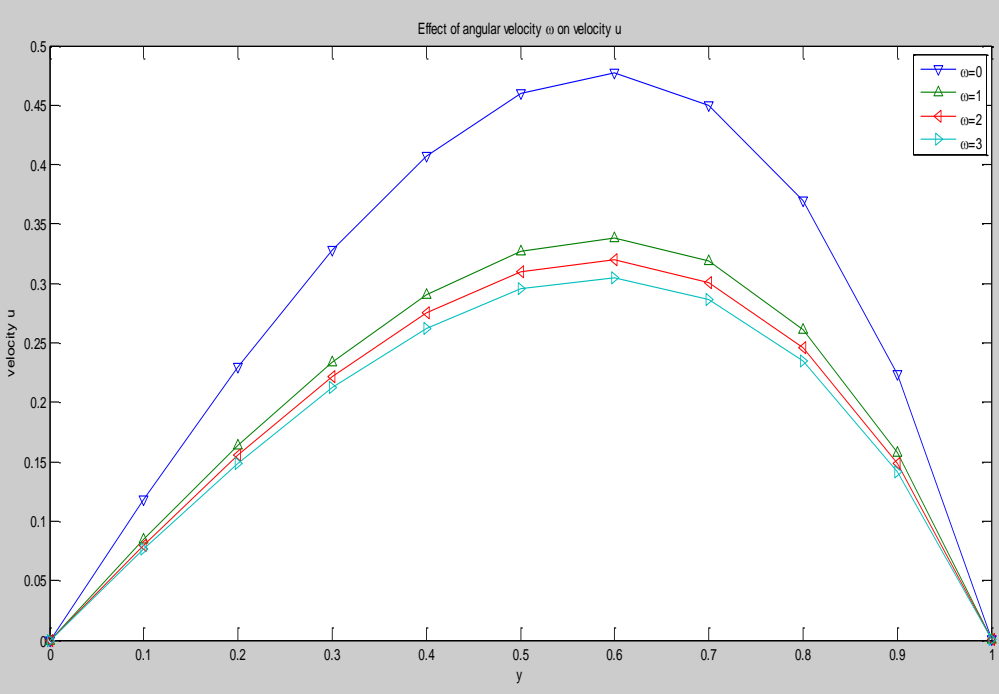

Figure 6. 


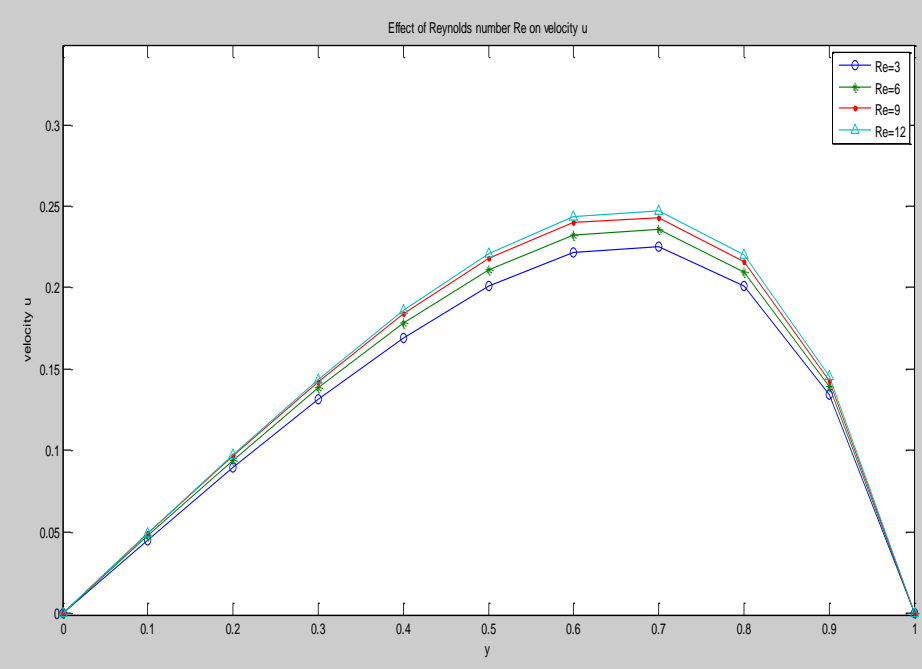

Figure 7.

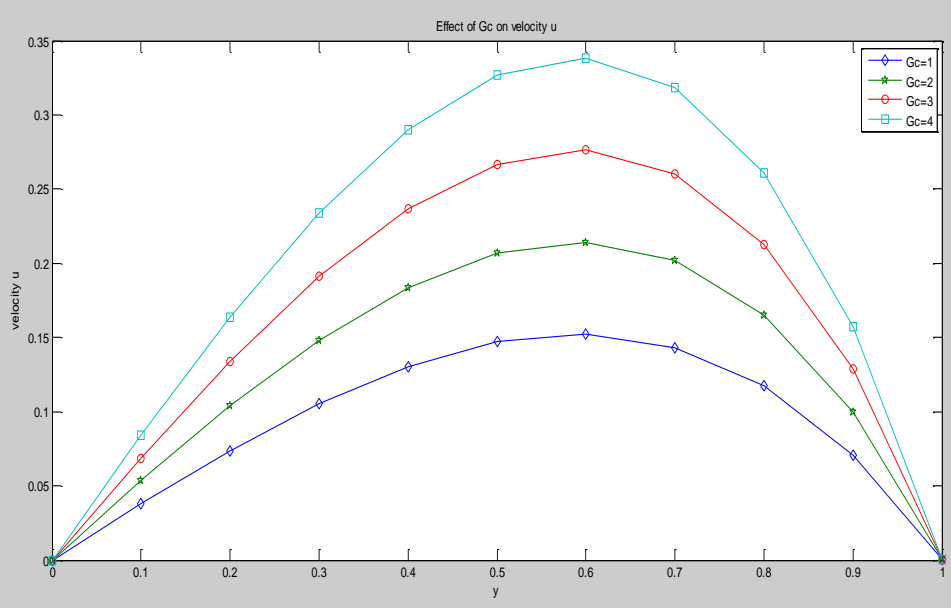

Figure 8.

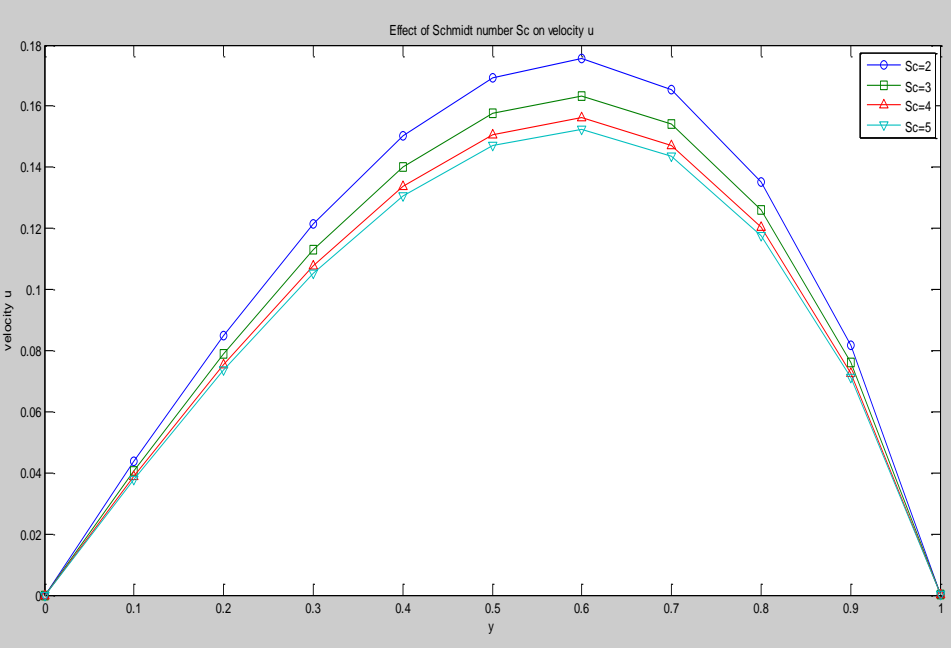

Figure 9. 


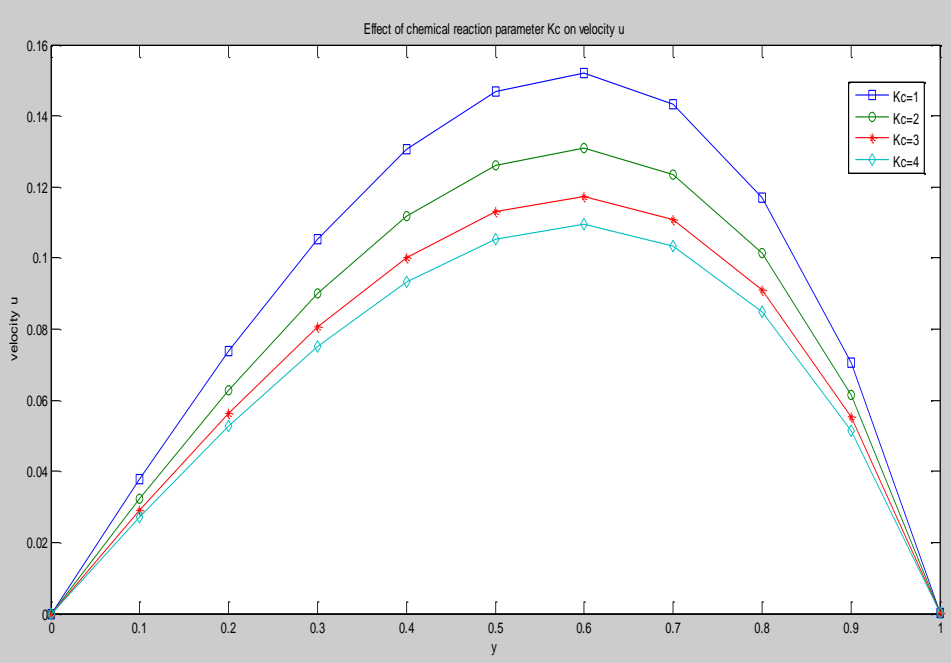

Figure 10.

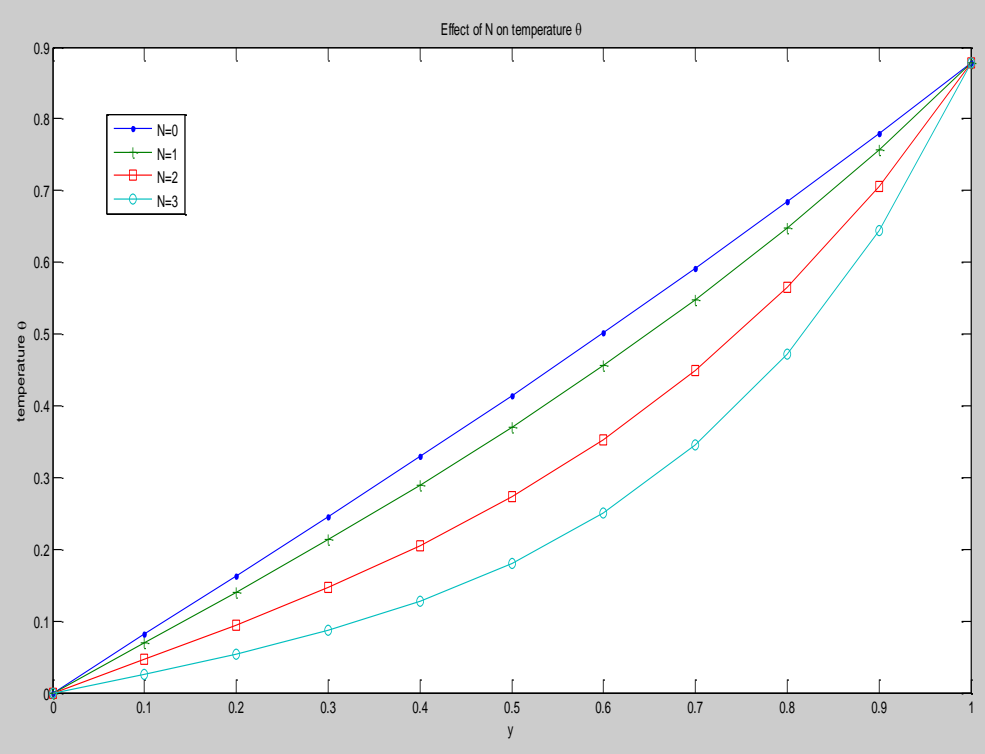

Figure 11.

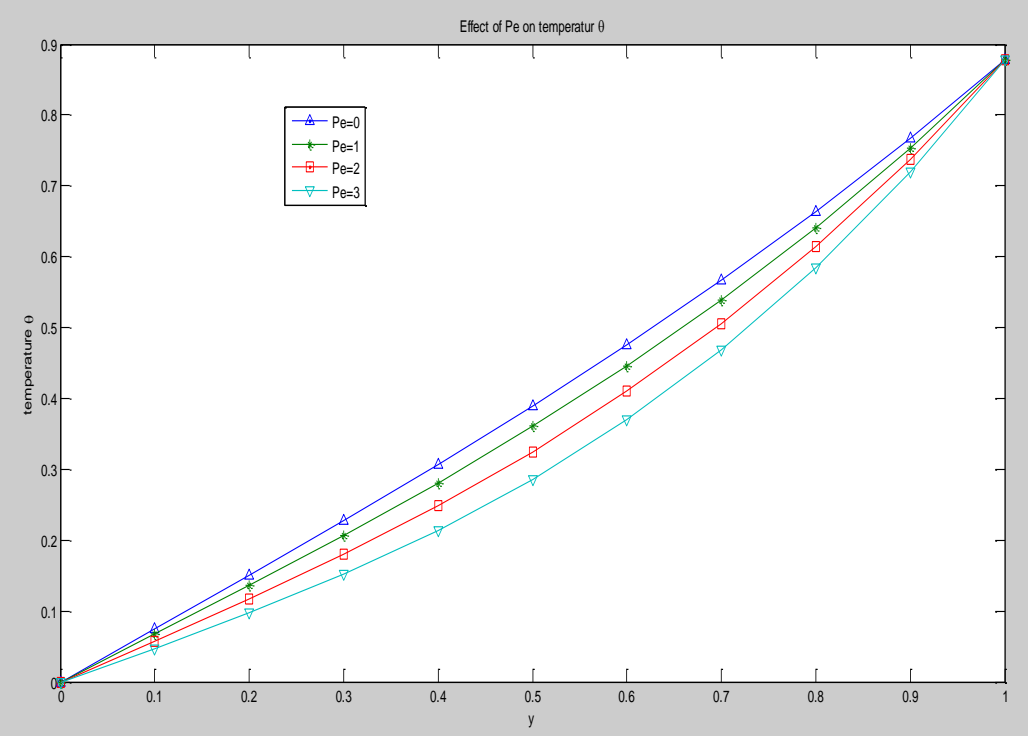

Figure 12. 


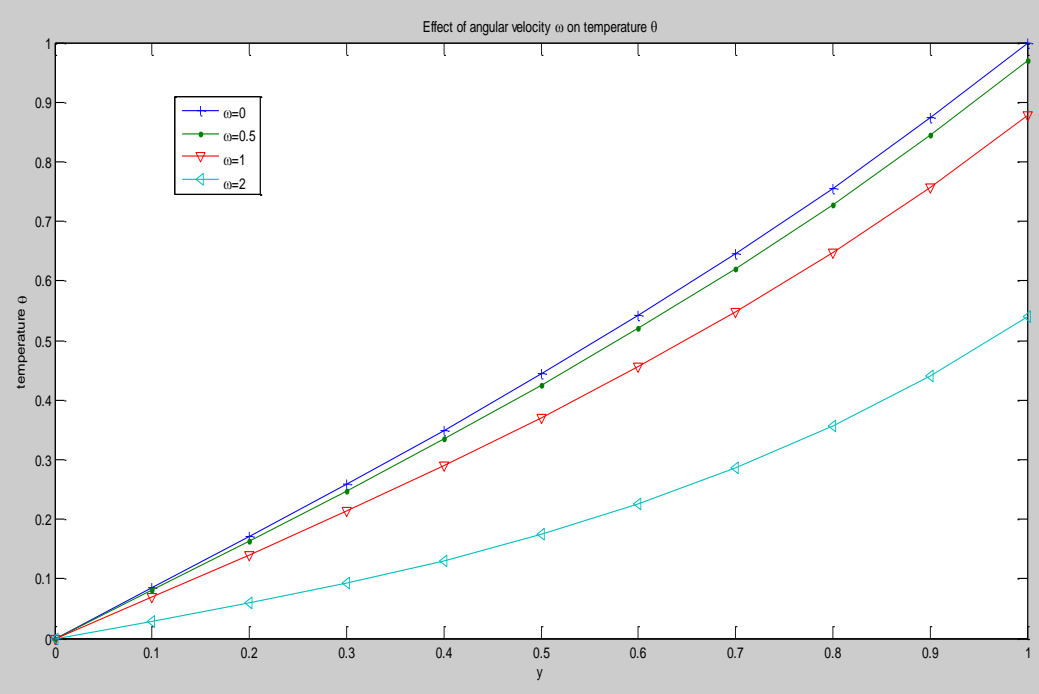

Figure 13.

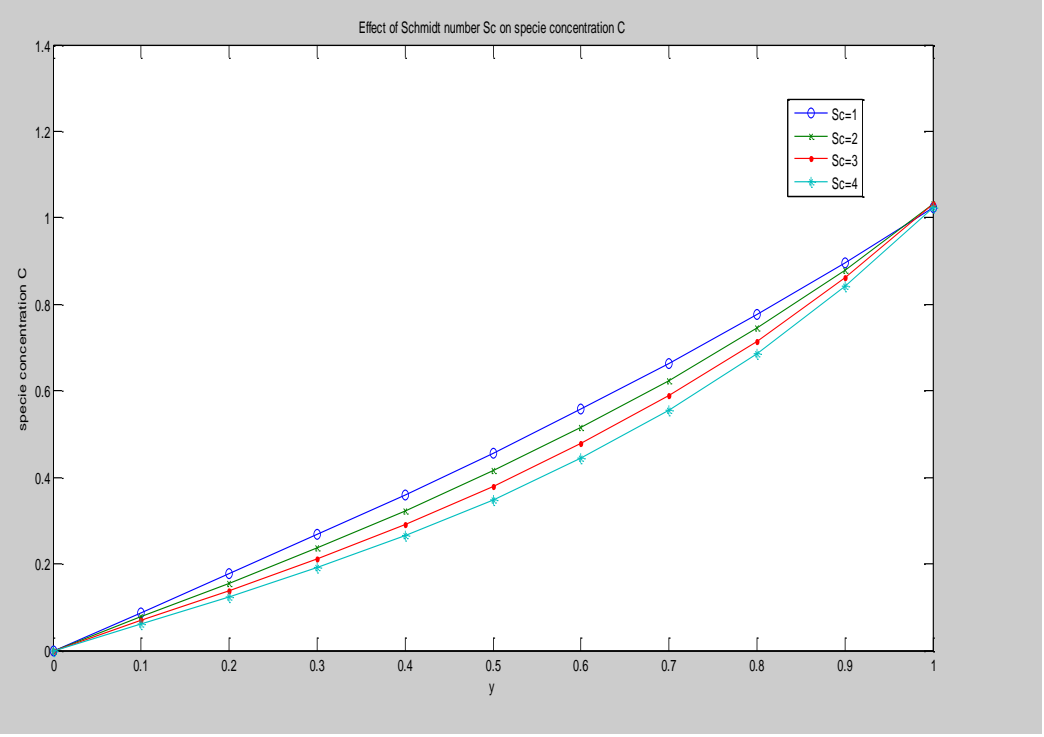

Figure 14

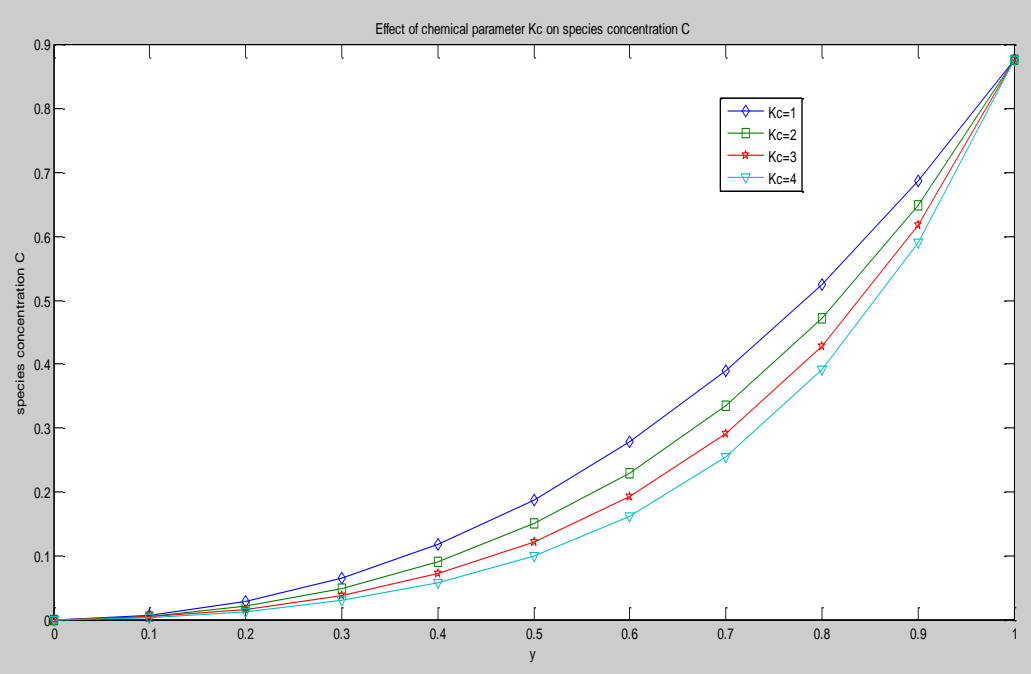

Figure 15. 


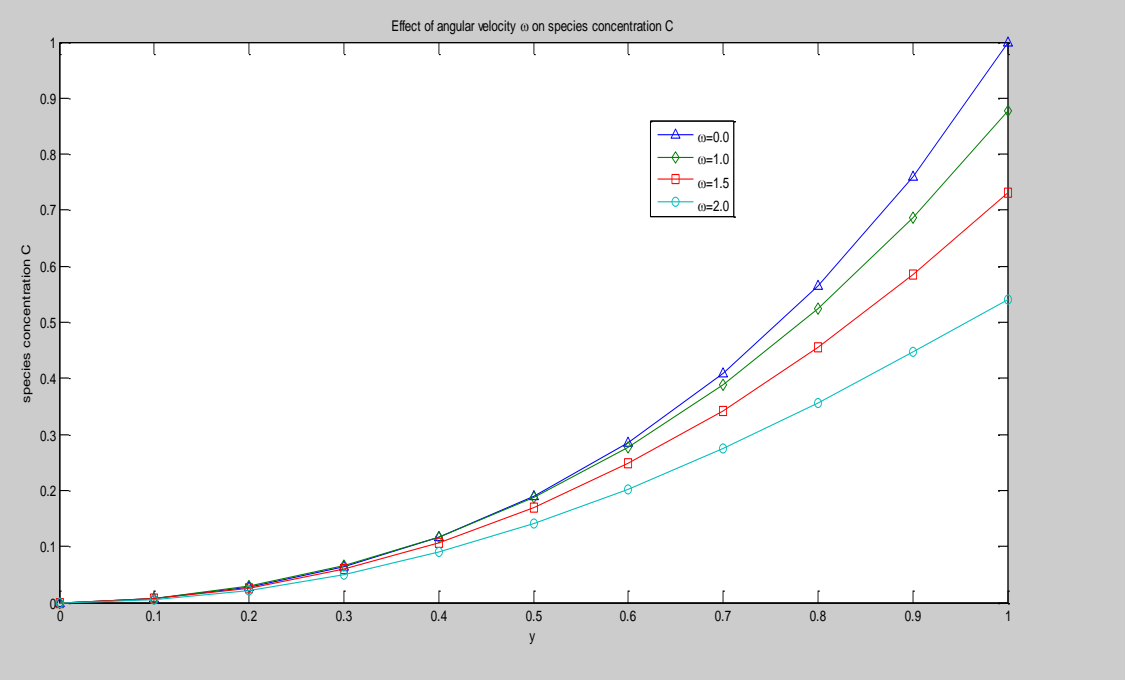

Figure 16.

Table 1. Variation of velocity $u$ with $y$ for different values of $P e$.

\begin{tabular}{|c|c|c|c|c|c|}
\hline$y$ & $P e=0.0$ & $P e=0.5$ & $P e=1.0$ & $P e=1.5$ & $P e=2.0$ \\
\hline 0.0 & 0.0000 & 0.0000 & 0.0000 & 0.0000 & 0.0000 \\
\hline 0.1 & 0.0465 & 0.0452 & 0.0446 & 0.0443 & 0.0440 \\
\hline 0.2 & 0.0905 & 0.0880 & 0.0867 & 0.0860 & 0.0854 \\
\hline 0.3 & 0.1293 & 0.1256 & 0.1237 & 0.1225 & 0.1216 \\
\hline 0.4 & 0.1602 & 0.1554 & 0.1529 & 0.1512 & 0.1494 \\
\hline 0.5 & 0.1803 & 0.1748 & 0.1716 & 0.1694 & 0.1674 \\
\hline 0.6 & 0.1866 & 0.1805 & 0.1769 & 0.1742 & 0.1716 \\
\hline 0.7 & 0.1756 & 0.1696 & 0.1657 & 0.1627 & 0.1598 \\
\hline 0.8 & 0.1437 & 0.1385 & 0.1349 & 0.1320 & 0.1290 \\
\hline 0.9 & 0.0867 & 0.0833 & 0.0809 & 0.0788 & 0.0767 \\
\hline 1.0 & 0.0000 & 0.0000 & 0.0000 & 0.0000 & 0.0000 \\
\hline
\end{tabular}

Table 2. Variation of skin friction $\tau_{0}$ and $\tau_{1}$ with different values of time $t$.

\begin{tabular}{|c|c|c|}
\hline$t$ & $\tau_{0}$ & $\tau_{1}$ \\
\hline 0.0 & 2.3655 & 2.8962 \\
\hline 0.1 & 2.3649 & 2.8975 \\
\hline 0.2 & 2.3643 & 2.8991 \\
\hline 0.3 & 2.3637 & 2.9007 \\
\hline 0.4 & 2.3629 & 2.9025 \\
\hline 0.5 & 2.3622 & 2.9044 \\
\hline 0.6 & 2.3163 & 2.9063 \\
\hline 0.7 & 2.3605 & 2.9084 \\
\hline 0.8 & 2.3586 & 2.9104 \\
\hline 0.9 & 2.3586 & 2.9125 \\
\hline 1.0 & 2.3577 & 2.9146 \\
\hline
\end{tabular}

Table 3. Variation of Nusselt numbers $N u_{0}$ and $N u_{1}$ with different values of time $t$.

\begin{tabular}{|c|c|c|}
\hline$t$ & $N u_{0}$ & $N u_{1}$ \\
\hline 0.0 & 0.8028 & 1.5233 \\
\hline 0.2 & 0.7713 & 1.4453 \\
\hline 0.4 & 0.7261 & 1.3496 \\
\hline 0.5 & 0.6989 & 1.2962 \\
\hline 1.0 & 0.5281 & 0.9966 \\
\hline 1.2 & 0.4500 & 0.8727 \\
\hline 1.5 & 0.3321 & 0.6978 \\
\hline 2.0 & 0.1590 & 0.4730 \\
\hline 5.0 & 0.1337 & 0.2997 \\
\hline 10.0 & 0.1219 & 0.1758 \\
\hline
\end{tabular}


Table 4. Variation of Sherwood number $S h_{0}$ and $S h_{1}$ with different values of time $t$.

\begin{tabular}{|c|c|c|}
\hline$t$ & $S h_{0}$ & $S h_{1}$ \\
\hline 0.0 & -1.4835 & -0.9527 \\
\hline 0.1 & -1.4854 & -0.9555 \\
\hline 0.2 & -1.4875 & -0.9589 \\
\hline 0.3 & -1.4901 & -0.9628 \\
\hline 0.4 & -1.4930 & -0.9672 \\
\hline 0.5 & -1.4961 & -0.9721 \\
\hline 0.6 & -1.4996 & -0.9774 \\
\hline 0.7 & -1.5033 & -0.9831 \\
\hline 0.8 & -1.5071 & -0.9891 \\
\hline 1.0 & -1.5111 & -0.9953 \\
\hline
\end{tabular}

\section{Summary and Conclusion}

In this section, we studied the effect of heat transfer on unsteady MHD oscillatory of Jeffrey fluid in a horizontal channel with chemical reaction.

The governing equations, that is, the momentum, energy and species equations have been written in dimensionless form.

A perturbation method has been employed to evaluate and solved the dimensionless velocityu, the dimensionless temperature $\theta$, the dimensionless species concentration $C$ skin frictions $\tau_{0}$ and $\tau_{1}$, Nusselt numbers $N u_{0}$ and $N u_{1}$ and Sherwood numbers $S h_{0}$ and $S h_{1}$.

The main findings are as follows:

i. Increase in Grashof number for heat transfer $\mathrm{Gr}$, Grashof number for mass transfer $\mathrm{Gc}$ and $\mathrm{Re}$ have accelerating effects on velocity of the flow field

ii. Decrease in material parameter $\lambda_{1}, H a, S c, K_{c}$ and $\omega$ accelerates the velocity of the flow.

iii. Peclet number retards the velocity of the flow field.

iv. Increase in radiation parameter $N, R e$ and $\omega$ retard the magnitude of temperature of the fluid flow.

v. Decrease in $S c, K c$ and $\omega$ increase the species concentration or the concentration boundary layer thickness of the flow field.

It is found that, the velocity $u$ increases with increase in $G r, G c, N$ and Re. The velocity also increases with decrease $\lambda_{1}, H a, S c, K_{c}$ and $\omega$. The velocity only decreases with increase $P e$. It is also observed that the temperature $\theta$ decreases with increase in $N, \operatorname{Re}$, and $\omega$.

The significant of the study shows that the velocity $u$ is more of Jeffrey fluid than that of Newtonian fluid. This study has the potentials applications in oil recovery, filtration systems and in the area of finding remedy for atherosclerosis and area of medicine

\section{References}

[1]. Aruna Kumari B., Ramakrishna Prasad K., Kavita K. (2012): Slip Effects on MHD Oscillatory Flow of Jeffrey Fluid in a Channel with Heat Transfer. Int. J. Math. Arch. 3 (8):2903 - 2911.

[2]. Asadulla M., Umar K., Nareed A., Raheela M. and Mohyud - Din S.T. (2013): MHD Flow of a Jeffrey Fluid in Converging and Diverging Channels. Int. J. Mod. Math. Sci. 6 (2): 92 - 106.

[3]. Aziz A. and Na T.Y. (1984): Perturbation Methods in Heat Transfer. Hemisphere, New York.

[4]. Badari Narayana C.H, Sreenadh S. and Devaki P. (2012): Oscillatory Flow of Jeffrey Fluid in Elastic tube of Variable Cross Section. Adv. Appl. Sci. Res. 3 (2): $671-677$.

[5]. Bharali A. and Borkakati A.K. (1980): The Flow and Heat Transfer between Two Horizontal Parallel Plates. Journal of the Physical Society of Japan. 49 (5): 2091.

[6]. Bharali A. and Borkakati A.K. (1980): The Heat Transfer in an Axisymmetric Flow between Two Parallel Porous Disk under the Effect of a Transverse Magnetic Field. Journal of the Physical Society of Japan. 52 (1): 6.

[7]. Bodosa G. and Borkakati A.K. (2003): MHD Couette Flow with Heat Transfer between Two Horizontal Plates in the Presence of Uniform Transverse Magnetic Field. Theor. Appl. Mech. 30 (1): 1 - 9.

[8]. Devika B., Satga Narayana P.V. and Venkatarmana S. (2013): MHD Oscillatory Flow of a Viscous Elastic Fluid in a Porous Channel with Chemical Reaction. Int. J. Eng. Sci. Inv. 2(2): $26-35$.

[9]. Israel - Cookey C., Nwaigwe C. (2010): Unsteady MHD Flow of a Radiating Fluid over a Moving Heated Porous Plate with Time - Dependent Suction. Am. J. Sci. Ind. Res. 1 (1): 88 - 95.

[10]. Israel - Cookey C., Omubo - Pepple V.B. and Tamunoberetonari I. (2010): On Steady Hydromagnetic Flow of a Radiating Viscous Fluid through a Horizontal Channel in a Porous medium. Am. J. Sci. Ind. Res. 1 (2): $303-308$.

[11]. Joseph K.M., Onwubuoya C., Daniel K.S., and Nonum E.O. (2013): MHD Forced Convection in a Horizontal Double - Passage with Uniform Wall Heat Flux. Int. J. Math. Stat. Stud. 1 (2): 30 - 40.

[12]. Kavita K., Pasad R.K. and Kumari B. A. (2012): Influence of Heat Transfer on MHD Oscillatory Flow of Jeffrey Fluid in a Channel. Adv. Appl. Sci. Res. 3 (4): 2312 - 2325.

[13]. Kim U.J. (2000): Unsteady MHD Convective Heat Transfer past a Semi - Infinite Vertical Porous Moving Plate with Variable Suction. Int. J. Eng. Sci. 38: $833-845$

[14]. Kumar A., Varshney C. L., Lal S. (2010):Perturbation Technique to Unsteady Periodic Flow of Viscous Fluid through a Planar Channel. J. Eng. Tech. Res. 2 (4): $73-81$. 
[15]. Kumar N., Jain T. and Gupta S. (2012): Unsteady MHD Free Convection Flow through Porous Medium Sandwiched between Viscous Fluids. Int. J. Ener. Tech. 4 (27): 1- 11

[16]. Maen Al - Rashdan (2012): Heat and Mass Fully - Developed Natural Viscous Flow with Chemical Reaction in Porous Medium. Adv. Theor. Mech. 5 (3): $93-112$.

[17]. Makinde O.D., Mhone P.Y. (2005): Heat Transfer to MHD Oscillatory Flow in a Channel Filled with Porous Medium. Romanian J. Physics. $50(9-10): 931-938$.

[18]. Pathak G. and Maheshwari C.H. (2006): Effects of Radiation on Unsteady Free Convection Flow Bounded by an Oscillating Plate with Variable Wall Temperature. Int. J. Appl. Mech. Eng. 11 (2): 371 - 382.

[19]. Rita C. and Das K.S. (2013): Visco - Elastic Unsteady MHD Flow between Two Horizontal Parallel Plates with Hall Current. TOSR J. Math. 5 (1): $20-28$.

[20]. Sharma S. and Deka R.K. (2012): Thermal Radiation and Oscillating Plate Temperature Effects on MHD Unsteady Flow past a Semi - Infinite Vertical Plate under Suction and Chemical Reaction. Int. J. Phys. Math. Sci. 2 (2): 33 - 52.

[21]. Sharma V.K., Singh J. Varshney N.K. and Gupta P.C.(2011): Numerical Steady of Two - Dimensional MHD Formed Stagnation - Point Flow in the Presence of Hall Current. Applied Math. Sci. 5 (30): 1473 - 1479

[22]. Siddiqui A.M., Farooq A.A., Haroon T. and Babcock B.S. (2013): A Variant of the Classical Von Karma Flow for Jeffrey Fluid. Appl. Math. Sci. 7 (2): $983-991$.

[23]. Singh P. and Gupta C.B. (2005): MHD Free Convective of Flow of Viscous Fluid through a Porous Medium Bounded by an Oscillating Porous Plate in Slip Flow Regime with Mass Transfer. Indian J. Theor. Physics 53 (2): $111-120$.

[24]. Sreenadh S., Devaki P. and Diwakar R. (2012): Unsteady Flow of Jeffrey Fluid in an Elastic Tube with Steno Sis .International Conference of Fluid Dynamics and Thermodynamics Technologies, IPCSIT Vol 33, IACSIT Press, Singapore.

[25]. Vineet K. S., Gupta P.C., Varshney N.K., Vineet K.S. (2012): MHD Effects on Free Convection Fluid Flow Past a Vertical Surface with Porous Medium and Radiation. Int. J. Math. Analysis, 6 (1):29 - 37. 